\title{
Effects of Maxwellian ions on Dust-acoustic Solitary Waves in Adiabatic Degenerate Plasmas and It's Instability
}

\author{
Nushrat Khan, Sharmin Yiasmin Swarna and Md. Masum Haider*
}

Department of Physics, Mawlana Bhashani Science and Technology University, Santosh, Tangail-1902, Bangladesh.

\author{
Received: October 11, 2017; Accepted: October 25, 2017; Published: November 23, 2017 \\ *Corresponding author: Md. Masum Haider, Department of Physics, Mawlana Bhashani Science and Technology University, Santosh, \\ Tangail-1902, Bangladesh; Email: masum.phy@gmail.com/masum@mbstu.ac.bd
}

\begin{abstract}
A rigorous theoretical investigation has been made on dust acoustic solitary waves in unmagnetized and magnetized degenerate plasmas with adiabatic pressure. Korteweg-de Vries (K-dV) equation have been derived for unmagnetized case as well as its solution. For the case of magnetized plasmas, Zakharov-Kuznetsov (ZK) equation, and its solution have been derived and studied its instability criterion and growth rate. It has been found that, the parametric regimes effects not only modify the basic properties of dust acoustic solitary waves and its instability criterion but also introduce some important new features.
\end{abstract}

Keywords: Maxwellian ions; Dust-acoustic Solitary Waves; Degenerate Plasmas; K-dV equation; ZK euation; Instability;

\section{Introduction}

The propagation of dust acoustic waves (DASW's) waves plays an important role in understanding the different behaviour of dust components or different waves phenomena in dusty plasmas which are omnipresent in laboratory, space and astrophysical plasma environments, such as cometary tails, planetary ring, interstellar medium etc. The dust grain changes the nature of the system including the creation of the new modes. In the recent years, many important and precious, theoretical and experimental investigation has been made on solitary waves. DASW's are one of the most significant non-linear affair in plasma method. Different properties such as amplitude, width etc. of the solitary waves can be modified by using different perturbation method. Bliokh and Yaroshenko studied the elecrostatic waves in dusty plasmas and applied their results in interpreting spoke-like structures in Saturn's rings (revealed by the voyager space mission)[1, 2]. Rao et.al, were the first to predict theoretically existence of extremely lower phase velocity DASW's in unmagnetized dusty plasmas whose constituent are inertial charged dust grains and Boltzmann distributed ions and electrons [3]. Roychoudhury and Mukherjee considered a two-component unmagnetized dusty plasma consisting of a negatively charged adiabatic dust fluid and an inertia-less isothermal ion fluid and investigated the effects of dust fluid temperature on large amplitude solitary waves by the pseudo-potential approach [4]. Sayed and Mamun assumed a dusty plasma containing the adiabatic dust fluid and non-adiabatic (isothermal) inertia-less electron and ion fluid and studied the effect of the dust fluid temperature on the DASW's by the reductive perturbation method $[5,6]$. Mendoza-Briceño et al, assumed a two-component dusty plasma containing the adiabatic dust fluid and non-adiabatic ions following the nonthermal distribution of Cairns et al and studied the effect of the dust fluid temperature on the DA solitary waves by the pseudo-potential approach [7-9].

The equation of state for degenerate electrons in such intersteller compact objects are mathematically explained by Chandrasekhar for two limits, namely non-relativistic and ultrarelativistic limits $[10,11]$. The degenerate electron equation of state of Chandrasekhar is $P_{e}$ is proportional to $N_{e}^{5 / 3}$ for the non-relativistic limit and $P_{e}$ is proportional to $N_{e}^{4 / 3}$ for the ultrarelativistic limit where $P_{e}$ is the degenerate electron pressure and $N_{e}$ is the degenerate electron number density. Mamun and Shukla considered an unmagnetized degenerate plasma without and with negatively charged stationary dust $[12,13]$. Mamun et al, studied the ion acoustic Solitary Waves (SW's) in the presence of an external magnetic field for ultra-relativistic degenerate electron-ion plasmas using the reductive perturbation technique [14]. Using the same technique Haider et al, also studied the obliquely propagating solitary structure with the presence of external magnetic field $[15,16]$. But the presence of heavy ions, which can be arbitrary charged, were not considered in the work of Mamun et al and Haider et al [14,15].

In the present work we have studied the nonlinear propagation of DA SW's in unmagnetized and magnetized degenerate plasmas with adiabatic pressure. We have studied both ultra-relativistic and non-relativistic case simultaneously using the generalized equation proposed by Haider [17]. To do this we have derived Korteweg-de Vries (K-dV) and Zakharov-Kuznetsov (ZK) equation by reductive perturbation method and find out the solution of it [6]. We have studied the instability criterion as well as it's growth rate. 


\section{Plasma Model}

We have studied the nonlinear propagation of DASW's in unmagnetized and magnetized degenerate plasmas for adiabatic situation containing

(i) inertial positive mobile dust component

(ii) inertialess degenerate electrons

(iii) inertialess degenerate positrons

(iv) stationary negative dust component

(v) inertial Maxwellian ions

At equilibrium, we have,

$$
n_{p d 0}+n_{i 0}+n_{p 0}=n_{n d 0}+n_{e 0}(1)
$$

where, $n_{p d 0}, n_{i 0}, n_{p 0}, n_{n d 0}, n_{e 0}$ are the positive dust, ion, positron, negative dust and electron number densities at equilibrium respectively.

\section{Solitary Waves in Unmagnetized Plasmas}

\section{Basic Equations}

The dynamics of such DASW's in one dimensional form and is given by the followings equations,

$$
\begin{gathered}
\frac{\partial n_{p d}}{\partial t}+\frac{\partial}{\partial x}\left(n_{p d} u_{p d}\right)=0 \\
\frac{\partial u_{p d}}{\partial t}+\left(u_{p d} \frac{\partial}{\partial x}\right) u_{p d}=-\frac{\partial \varphi}{\partial x}-\left(\frac{\sigma_{d}}{n_{p d}}\right) \frac{\partial}{\partial x} p_{p d} \\
\frac{\partial p_{p d}}{\partial t}+\left(u_{p d} \frac{\partial}{\partial x}\right) p_{p d}+\alpha p_{p d} \frac{\partial}{\partial x} u_{p d}=0 \\
\frac{\partial^{2} \varphi}{\partial x^{2}}=\left(\mu_{e} n_{e}-\mu_{p} n_{p}-n_{p d}-\mu_{i} n_{i}+\mu_{n d} n_{n d}\right)
\end{gathered}
$$

where, $n_{p d}$ is the positive dust number density normalized by its equilibrium value $n_{p d 0}, n_{n d}$ is the negative dust number density normalized by its equilibrium value $n_{n d 0}, n_{p}$ is the positron number density normalized by its equilibrium value $n_{p 0^{\prime}} n_{i}$ is the ion number density normalized by its equilibrium value $n_{i 0}, n_{e}$ is the electron number density normalized by its equilibrium value $n_{e 0}, u_{p d}$ is the dust fluid speed normalized by $C_{p d}=\left(m_{n d} c^{2} / m_{p d}\right)^{1 / 2}$, $m_{p d}$ is the rest mass of positive dust, $m_{n d}$ is the mass of negative dust and $C$ being the speed of light. $\varphi$ is the DA electrostatic wave potential normalized by $\left(m_{p d} C_{p d}^{2} / z_{d} e\right)$, with $z_{d}$ is the number of positive charge residing on the positive charged dust and $e$ being the magnitude of unit charge. $\alpha$ is the adiabatic index. $\sigma_{d}$ is the ratio of the positive dust temperature to negative dust temperature. $\mu_{e}=n_{e 0} / n_{p d 0}, \quad \mu_{p}=n_{p 0} / n_{p d 0}, \quad \mu_{i}=n_{i 0} / n_{p d 0}$ and $\mu_{n d}=n_{n d 0} / n_{p d 0}$
Here the space variables are normalized by Debye radius

$\lambda_{d}=\left(m_{p d} c^{2} / 4 \pi n_{d 0} z_{d}^{2} e^{2}\right)^{1 / 2}$ and time variable $(t)$ is normalized by $\omega_{p d}^{-1}=\left(m_{p d} / 4 \pi n_{p d 0} z_{d}^{2} e^{2}\right)^{1 / 2}$.

Non-thermal distributed ions can be represented by,

$$
n_{i}=\left(1-\varphi+\frac{1}{2} \varphi^{2}\right)
$$

According to Haider the value of $n_{e}$ and $n_{p}$ can be express respectively as $[18,19]$,

$$
\begin{aligned}
& n_{e}=\left(1+\frac{\gamma-1}{\beta} \varphi\right)^{\frac{1}{\gamma-1}} \\
& \text { and } \quad n_{p}=\left(1-\frac{\gamma-1}{\beta} \varphi\right)^{\frac{1}{\gamma-1}}
\end{aligned}
$$

where, $\beta=\left(\frac{K}{E}\right) n_{0}^{(\gamma-1)}$ where, $K=\frac{1}{18(\gamma-1)}\left(\frac{3 \Delta^{3}}{\pi}\right)^{(\gamma-1)} E$ with

$\Lambda\left(=\frac{h}{m_{p d} c}\right)$ is the Compton wavelength normalized by Debye radious $\left(\lambda_{d}\right), E=m_{p d} c^{2}$ and $n_{0}$ be the number density of plasma particle. $\gamma=4 / 3$ for ultra-relativistic case and $\gamma=5 / 3$ for nonrelativistic case.

\section{K-dV Equation}

We now follow the reductive perturbation method and construct a weakly nonlinear theory for the DA waves with small but finite amplitude, which leads to a scaling of the independent variables through the stretched coordinates as $[6,18,19]$,

$$
\begin{gathered}
Z=\varepsilon^{1 / 2}\left(x-v_{p} t\right) \\
\tau=\varepsilon^{3 / 2} t
\end{gathered}
$$

where, $\varepsilon$ is a smallness parameter measuring the weakness of the dispersion, $v_{p}$ is the nonlinear wave phase velocity. We can expand the perturbed quantities $n_{p d}, u_{p d}, p_{p d}$ and $\phi$ about their equilibrium values in powers of $\varepsilon$,

$$
\begin{aligned}
& n_{p d}=1+\varepsilon n_{p d}^{(1)}+\varepsilon^{2} n_{p d}^{(2)}+\ldots \\
& u_{p d}=0+\varepsilon u_{p d}^{(1)}+\varepsilon^{2} u_{p d}^{(2)}+\ldots \\
& \varphi=0+\varepsilon \varphi^{(1)}+\varepsilon^{2} \varphi^{(2)}+\ldots \\
& p_{p d}=1+\varepsilon p_{p d}^{(1)}+\varepsilon^{2} p_{p d}^{(2)}+\ldots
\end{aligned}
$$

Using the stretched coordinates and equations (11)-(14) in equations (2)-(5); and equating the coefficients of $\varepsilon^{3 / 2}$ from the continuity and momentum equation and coefficients of $\varepsilon$ from Poisson's equation and rearranging the parameters one can obtain the linear dispersion relation for the DASW's 


$$
v_{p}=\sqrt{\frac{1}{\left(\frac{1}{\beta} \mu_{e}+\frac{1}{\beta} \mu_{p}+\mu_{i}\right)}+\alpha \sigma_{d}}
$$

It can be seen from equation (15) that the presence of the Maxwellian ions significantly modifies the linear dispersion relation.

Equating the next higher order co-efficient of $\varepsilon$ from above equations and using the parameters we can finally obtain a K-dV equation describing the nonlinear propagation of the DASW's in the dusty plasma

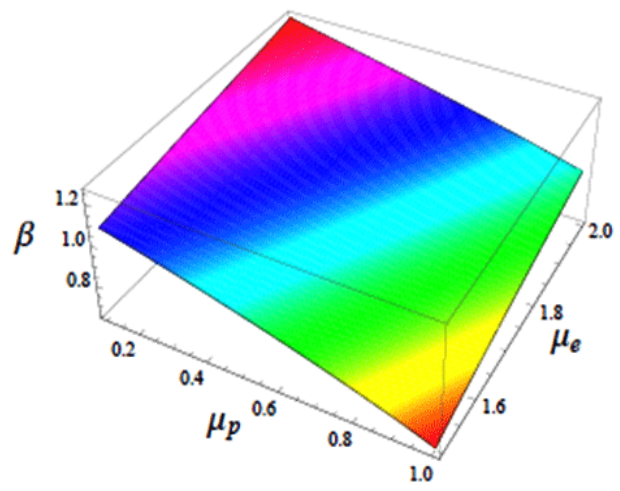

Figure 1: $A=0$ graph, showing the variation of $\beta$ with $\mu_{e}$ and $\mu_{p}$ for the values of $\alpha=0.5, \mu_{i}=0.4$ and $\gamma=5 / 3$

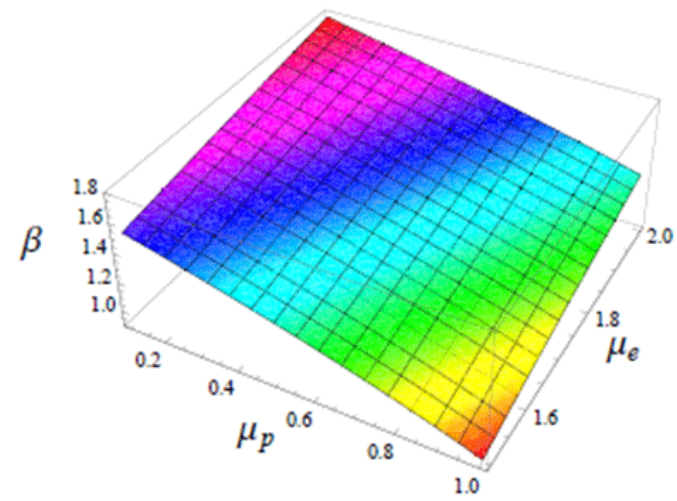

Figure 2: $A=0$ graph, showing the variation of $\beta$ with $\mu_{e}$ and $\mu_{p}$ for the values of $\alpha=0.5, \mu_{i}=0.4$ and $\gamma=4 / 3$

$$
\frac{\partial \phi^{(1)}}{\partial \tau}+A \phi^{(1)} \frac{\partial \phi^{(1)}}{\partial Z}+B \frac{\partial^{3} \phi^{(1)}}{\partial Z^{3}}=0
$$

where,

$$
\begin{gathered}
A=\left[\mu_{i}-\left(\mu_{e}-\mu_{p}\right)\left(\frac{2-\gamma}{\beta^{2}}\right)\right] \frac{\left(v_{p}^{2}-\alpha \sigma_{d}\right)^{2}}{2 v_{p}} \\
B=\frac{\left(v_{p}^{2}-\alpha \sigma_{d}\right)^{2}}{2 v_{p}}
\end{gathered}
$$

\section{Solution of K-dV Equation}

The stationary solution of this K-dV equation can be obtained by transforming the independent variables $\xi$ and $\tau$ to $\xi=Z-u_{0} \tau$, $\tau=t$, where $u_{0}$ is a constant solitary wave velocity. For simplicity, we have write $\varphi^{(1)}$ as $\varphi$. Now using the appropriate boundary conditions for localized disturbances, viz.

$$
\varphi^{(1)} \rightarrow 0,\left(d \varphi^{(1)} / d \xi\right) \rightarrow 0,\left(d^{2} \varphi^{(1)} / d \xi^{2}\right) \rightarrow 0
$$

at $\xi \rightarrow \pm \infty$. Thus, one can express the stationary solution of this $\mathrm{K}-\mathrm{dV}$ equation as

$$
\phi=\phi_{m} \operatorname{sech}^{2}[\xi / \Delta]
$$

where $\varphi_{m}=\left(3 u_{0} / A\right)$ is the amplitude and $\Delta=\sqrt{\frac{4 B}{u_{0}}}$ is the width of the solitary waves. The value of $A$ and $B$ are shown in (17) and (18) respectively.

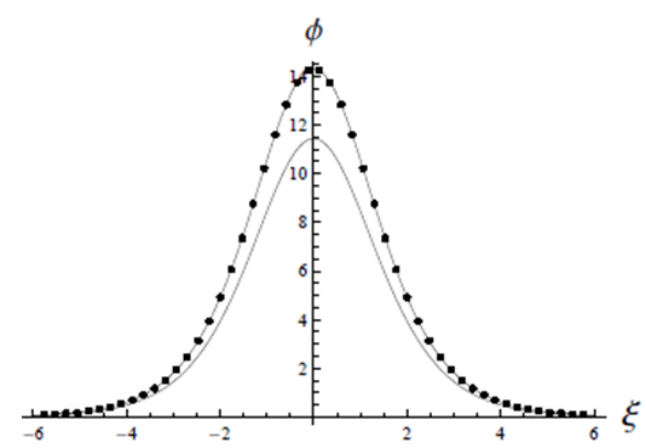

Figure 3: Variation of $\varphi$ with $\xi$ for the values of $\alpha=0.5, \mu_{p}=1$ $\mu_{i}=0.4, \mu_{e}=1.2, u_{0}=0.1, \sigma_{d}=1, \beta=1 \gamma=5 / 3$ (solid curve) and $\gamma=4 / 3$ (dotted curve).

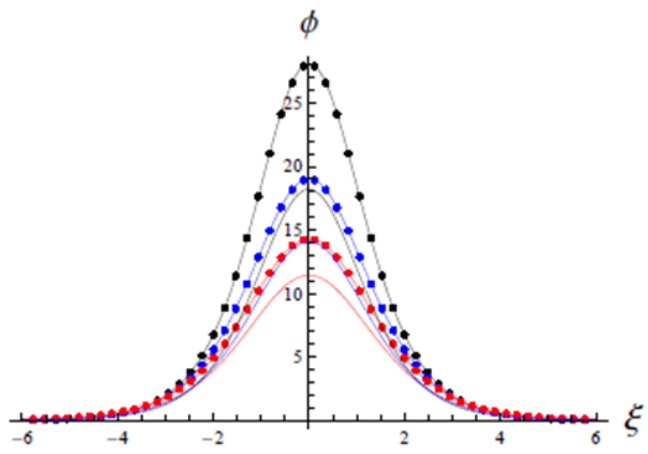

Figure 4: Variation of $\varphi$ with $\xi$ for the values of $\alpha=0.5, \mu_{p}=1$, $\mu_{i}=0.4, \mu_{e}=1.2, u_{0}=0.1, \sigma_{d}=1, \beta=0.8$ (Black), $\beta=0.9$ (Blue) and $\beta=1$ (Red). Here dotted curve represented ultra-relativistic $(\gamma=4 / 3)$ case and solid curve represented non-relativistic $(\gamma=5 / 3)$ case.

\section{Solitary Waves in Magnetized Plasmas}

\section{Basic Equations}

We have been considered that, there an external static magnetic field present $B_{0}$ acting along the z-direction $B_{0}=\hat{k} B_{0}$ ) 
where $\hat{k}$ is the unit vector along the z-direction, so that the ions and dusts are moving along the magnetic field direction.

The dynamics of such DASW's in three dimensional form and is given by the followings equations,

$$
\begin{gathered}
\frac{\partial n_{p d}}{\partial t}+\nabla \cdot\left(n_{p d} u_{p d}\right)=0 \\
\frac{\partial u_{p d}}{\partial t}+\left(u_{p d} \cdot \nabla\right) u_{p d}=-\mathrm{v} \cdot \varphi+\omega_{c d}\left(u_{p d} \times \hat{k}\right) \\
-\left(\frac{\sigma_{d}}{n_{p d}}\right) \nabla p_{p d} \\
\frac{\partial p_{p d}}{\partial t}+\left(u_{p d} \cdot \nabla\right) p_{p d}+\alpha p_{p d} \nabla \cdot u_{p d}=0 \\
\nabla^{2} \varphi=\left(\mu_{e} n_{e}-\mu_{p} n_{p}-n_{p d}-\mu_{i} n_{i}+\mu_{n d} n_{n d}\right)
\end{gathered}
$$

Here, $\omega_{c d}$ is the positive dust cyclotron frequency $\left(z_{d} e B_{0} / m_{p d 0} c\right)$ normalized by

$$
\omega_{p d}=\left(\frac{4 \pi n_{p d 0} z_{d}^{2} e^{2}}{m_{p d}}\right)^{\frac{1}{2}}
$$

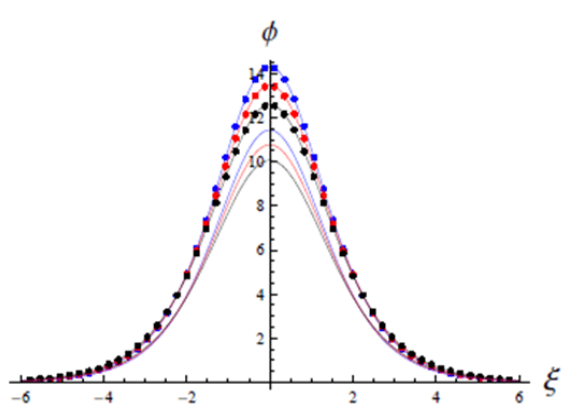

Figure 5: Variation of $\varphi$ with $\xi$ for the values of $\beta=1, \mu_{p}=1, \mu_{i}=0.4, \mu_{e}=1.2, u_{0}=0.1$, $\sigma_{d}=1, \alpha=0.3$ (Black), $\alpha=0.4$ (Red) and $\alpha=0.5$ (Blue). Here dotted curve represented ultrarelativistic $(\gamma=4 / 3)$ case and solid curve represented non-relativistic $(\gamma=5 / 3)$ case.

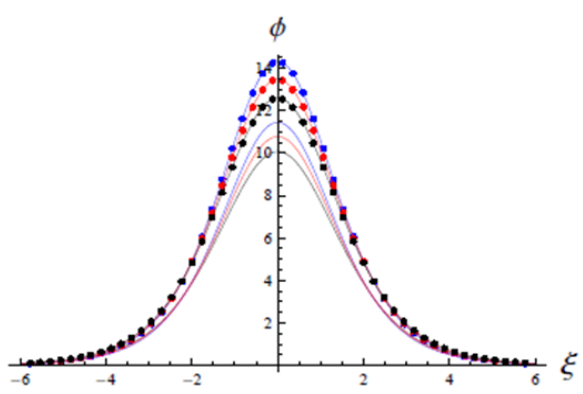

Figure 6: Variation of $\varphi$ with $\xi$ for the values of $\beta=1, \alpha=0.5, \mu_{p}=1, \mu_{i}=0.4, u_{0}=0.1$ $\mu_{e}=1.2, \sigma_{d}=0.6$ (Black), $\sigma_{d}=0.8$ (Red) and $\sigma_{d}=1$ (Blue). Here dotted curve representec ultra-relativistic $(\gamma=4 / 3)$ case and solid curve represented non-relativistic $(\gamma=5 / 3)$ case.

\section{Zakharov-Kuznetsov Equation}

To derive the Zakharov-Kuznetsov equation, which is known as ' $\mathrm{K}-\mathrm{dV}$ equation in three dimensions', we first introduce the following stretched coordinates

$$
\begin{aligned}
& X=\varepsilon^{1 / 2} x \\
& Y=\varepsilon^{1 / 2} y \\
& Z=\varepsilon^{1 / 2}\left(z-v_{p} t\right) \\
& \tau=\varepsilon^{3 / 2} t
\end{aligned}
$$

We can expand the perturbed quantities

$n_{p d}, u_{p d x}, u_{p d y}, u_{p d z}, p_{p d}$ and $\varphi$ about their equilibrium values in powers of $\varepsilon$

$$
\begin{gathered}
n_{p d}=1+\varepsilon n_{p d}^{(1)}+\varepsilon^{2} n_{p d}^{(2)}+\ldots \\
u_{p d x}=0+\varepsilon^{3 / 2} u_{p d x}^{(1)}+\varepsilon^{2} u_{p d x}^{(2)}+\ldots \\
u_{p d y}=0+\varepsilon^{3 / 2} u_{p d y}^{(1)}+\varepsilon^{2} u_{p d y}^{(2)}+\ldots \\
u_{p d z}=0+\varepsilon u_{p d z}^{(1)}+\varepsilon^{2} u_{p d z}^{(2)}+\ldots \\
\varphi=0+\varepsilon \varphi^{(1)}+\varepsilon^{2} \varphi^{(2)}+\ldots \\
p_{p d}=1+\varepsilon p_{p d}^{(1)}+\varepsilon^{2} p_{p d}^{(2)}+\ldots
\end{gathered}
$$

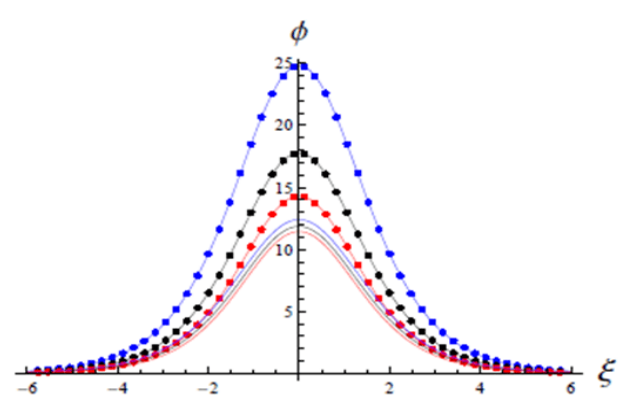

Figure 7: Variation of $\varphi$ with $\xi$ for the values of $\beta=1, \alpha=0.5, \mu_{i}=0.4, u_{0}=0.1, \mu_{e}=1.2$ $\sigma_{d}=1, \mu_{p}=0.8$ (Blue), $\mu_{p}=0.9$ (Black) and $\mu_{p}=1$ (Red). Here dotted curve represented ultrarelativistic $(\gamma=4 / 3)$ case and solid curve represented non-relativistic $(\gamma=5 / 3)$ case.

Next, substituting eqs. (24)-(33) into eqs. (20)-(23) and obtain the lowest order equations of (20)-(23) which in turn can be solved as (15), the linear dispersion relation for DASW's. This implies that linear dispersion relation for unmagnetized and magnetized situations are same. Magnetic field does not effect on the linear dispersion relation.

Equating the next higher order co-efficient of $\varepsilon$ from above equations and by the use of parameters we can finally obtained ZK equation describing the nonlinear propagation of the DA SW's in the dusty plasma 


$$
\begin{gathered}
\frac{\partial \varphi^{(1)}}{\partial \tau}+P Q \varphi^{(1)} \frac{\partial \varphi^{(1)}}{\partial Z} \\
+\frac{1}{2} P \frac{\partial}{\partial Z}\left[\frac{\partial^{2}}{\partial Z^{2}}+R\left(\frac{\partial^{2}}{\partial X^{2}}+\frac{\partial^{2}}{\partial Y^{2}}\right)\right] \varphi^{(1)}=0
\end{gathered}
$$

where,

$$
\begin{gathered}
P=\frac{\left(v_{p}^{2}-\alpha \sigma_{d}\right)^{2}}{v_{p}} \\
Q=\frac{1}{2}\left[\mu_{i}-\left(\mu_{e}-\mu_{p}\right)\left(\frac{2-\gamma}{\beta^{2}}\right)\right] \\
R=1+\frac{v_{p}^{4}}{\omega_{c d}^{2}\left(v_{p}^{2}-\alpha \sigma_{d}\right)^{2}}
\end{gathered}
$$

Equation (34) is a ZK equation for adiabatic DASW's with Maxwellian ions, degenerate electrons and positrons.

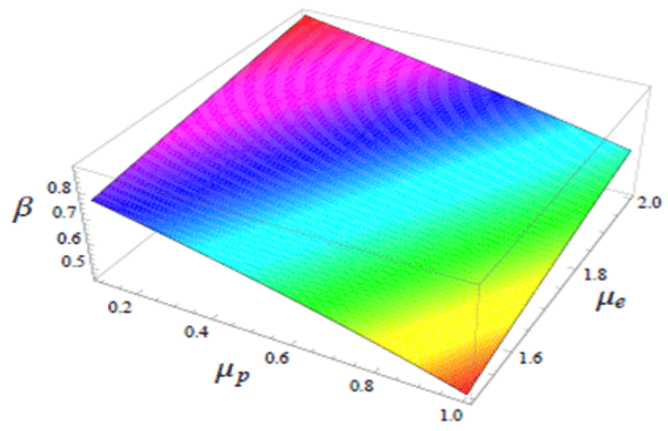

Figure 8: $P=0$ graph, showing the variation of $\beta$ with $\mu_{e}$ and $\mu_{p}$ for the values of $\alpha=0.5$, $\mu_{t}=0.4$ and $\gamma=5 / 3$

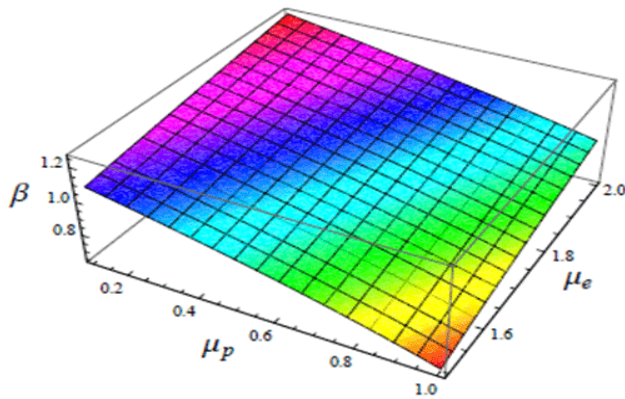

Figure 9: $P=0$ graph, showing the variation of $\beta$ with $\mu_{e}$ and $\mu_{p}$ for the values of $\alpha=0.5$ $\mu_{i}=0.4$ and $\gamma=4 / 3$.

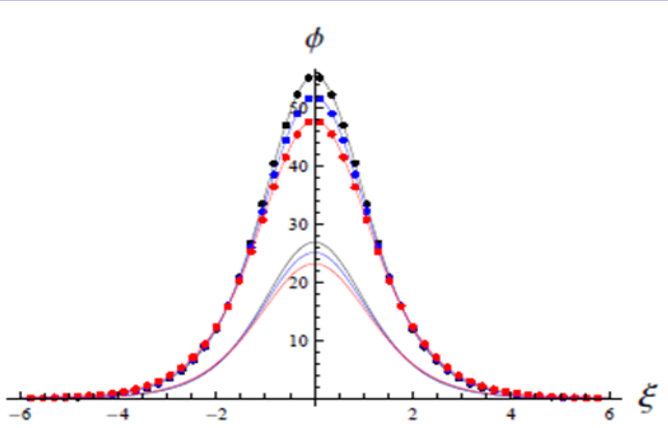

Figure 10: Variation of $\varphi$ with $\xi$ for the values of $\mu_{p}=1, \mu_{i}=0.4, \mu_{e}=1.2, \sigma_{d}=1, \omega_{c d}=0.3$, $\delta=20^{\circ}, \beta=0.7, u_{0}=0.1, \alpha=0.3$ (Red), $\alpha=0.4$ (Blue) and $\alpha=0.5$ (Black). Here dotted curve represented ultra-relativistic $(\gamma=4 / 3)$ case and solid curve represented non-relativistic $(\gamma=5 / 3$ ) case.

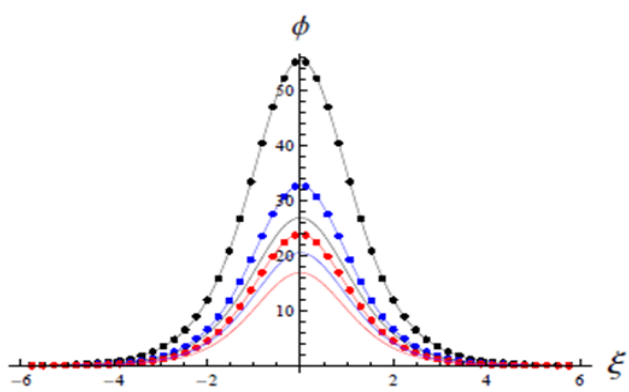

Figure 11: Variation of $\varphi$ with $\xi$ for the values of $\alpha=0.5, \beta=0.7, u_{0}=0.1, \mu_{p}=1, \omega_{c d}=0.3$, $\delta=20^{\circ}, \mu_{e}=1.2, \sigma_{d}=1, \mu_{i}=0.4$ (Black), $\mu_{i}=0.5$ (Blue) and $\mu_{i}=0.6$ (Red). Here dotted curve represented ultra-relativistic ( $\gamma=4 / 3)$ case and solid curve represented non-relativistic ( $\gamma=5 / 3$ ) case.

\section{Solution of Zakharov-Kuznetsov Equation}

The stationary solution (for simplicity, we have write $\varphi^{(1)}$ as $\varphi$ ) of this ZK equation as,

$$
\varphi(\xi)=\varphi_{m} \operatorname{sech}^{2}(k \xi)
$$

Where $\varphi_{m}=\left[\frac{3 u_{0}}{\delta_{1}}\right]$ is the amplitude and $k=\sqrt{\frac{u_{0}}{4 \delta_{2}}}$ is the inverse of the width $(\triangle)$ of the SW's respectively; with

$\delta_{1}=P Q \cos \delta$ and $\delta_{2}=\frac{1}{2} P\left(\cos ^{3} \delta+R_{\sin ^{2}}^{2} \delta \cos \delta\right), \delta$ being the propagation angle of SW's.

The $A=0(P=0)$ surface plots are shown for non-relativistic and ultra-relativistic case in absence (presence) of magnetic field are shown in figure 1 and 2 ( 8 and 9) respectively. From these figures we find that the SW's have positive potentials above the surface and negative potentials below the surface. Figure 3 shows the variation of $\varphi$ with $\xi$ for ultra-relativistic and non-relativistic cases. Figure 4, 5, 6 and 7 showing the variation of $\varphi$ with $\xi$ respectively for the different value of $\alpha, \sigma_{d}, \beta$ and $\mu_{p}$. All of these figure shows the variation of amplitude and width for ultrarelativistic and non-relativistic cases in the absence of magnetic field. Figure 10, 11, 12, 13, 14 and 15 showing the variation of $\varphi$ with $\xi$ for the value of $\alpha, \mu_{i}, \delta, \mu_{e}, \sigma_{d}$ and $\beta$ respectively in the presence of magnetic field. 


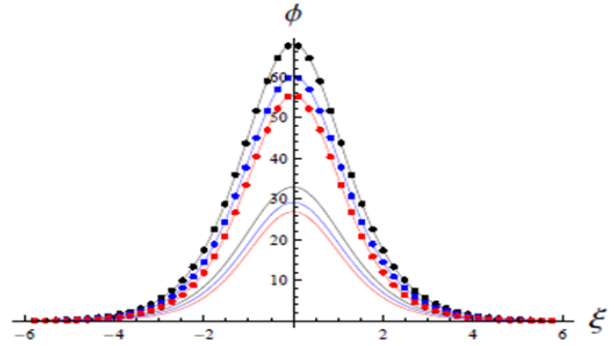

Figure 12: Variation of $\varphi$ with $\xi$ for the values of $\beta=0.7, \alpha=0.5, u_{0}=0.1, \mu_{p}=1, \mu_{t}=0.4$, $\mu_{e}=1.2, \sigma_{d}=1, \omega_{c d}=0.3, \delta=20^{\circ}$ (Red), $\delta=30^{\circ}$ (Blue) and $\delta=40^{\circ}$ (Black). Here dotted curve represented ultra-relativistic $(\gamma=4 / 3)$ case and solid curve represented non-relativistic $(\gamma=5 / 3$ ) case.

\section{Instability}

We now study the instability of the opaquely propagating solitary waves, discussed in the previous section, by the method of small- $k$ perturbation expansion (22-28). We first assume that separate $\varphi$ as a function of $\psi_{0}$ and $\psi$ as

$$
\varphi=\psi_{0}(Z)+\psi(\xi, \zeta, \eta, t)
$$

Where $\xi, \zeta, \eta$ and $t$ are components of new reference frame. For a long-wavelength plane wave perturbation in a direction with direction cosines $\left(l_{\zeta}, l_{\eta}, l_{\xi}\right), \psi$ is given by

$$
\psi=\phi(Z) e^{i\left[k\left(l_{\zeta} \zeta+l_{\eta} \eta+l_{\xi} Z\right)-\omega t\right]}
$$

in which $l_{\zeta}^{2}+l_{\eta}^{2}+l_{\xi}^{2}=1, k$ is the wave constant, $\omega$ is the angular frequency of the waves and $\phi$ is the amplitude of the waves.

For small $k, \phi(Z)$ and $\omega$ can be expanded as

$$
\begin{gathered}
\phi(Z)=\phi_{0}(Z)+k \phi_{1}(Z)+k^{2} \phi_{2}(Z)+\cdots \\
\omega=k \omega_{1}+k^{2} \omega_{2}+\cdots
\end{gathered}
$$

Doing some mathematical analysis we arrive at the following dispersion relation:

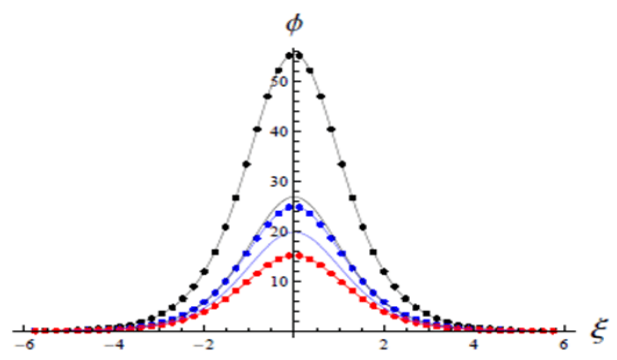

Figure 13: Variation of $\varphi$ with $\xi$ for the values of $\beta=0.7, \sigma_{d}=1, u_{0}=0.1, \omega_{c d}=0.3, \delta=20^{0}$, $\alpha=0.5, \mu_{p}=1, \mu_{i}=0.4, \mu_{s}=1$ (Red), $\mu_{e}=1.1$ (Blue) and $\mu_{e}=1.2$ (Black). Here dotted curve represented ultra-relativistic $(\gamma=4 / 3)$ case and solid curve represented non-relativistic $(\gamma=5 / 3$ ) case.

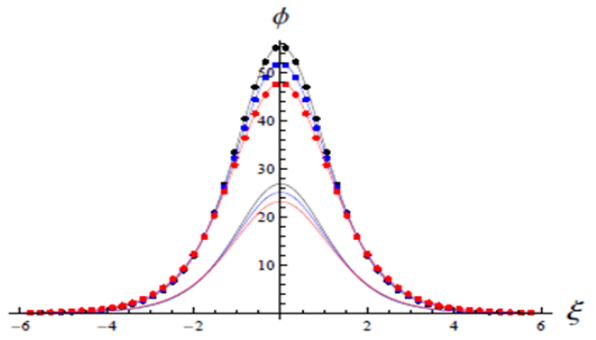

Figure 14: Variation of $\varphi$ with $\xi$ for the values of $\beta=0.7, \omega_{c d}=0.3, \delta=20^{\circ}, \alpha=0.5, u_{0}=0.1$ $\mu_{p}=1, \mu_{l}=0.4, \mu_{e}=1.2, \sigma_{d}=0.6$ (Red), $\sigma_{d}=0.8$ (Blue) and $\sigma_{d}=1$ (Black). Here dotted curve represented ultra-relativistic $(\gamma=4 / 3)$ case and solid curve represented non-relativistic $(\gamma=5 / 3$ ) case.

$$
\omega_{1}=\Omega-l_{\xi} U_{0}+\left(\Omega^{2}-\Upsilon\right)^{1 / 2}
$$

where

$$
\begin{aligned}
& \Omega=\frac{2}{3}\left(\psi_{m} \mu_{1}-2 \mu_{2} \kappa^{2}\right) \\
& \Upsilon=\frac{16}{45}\left(\psi_{m}^{2} \mu_{1}^{2}-3 \psi_{m} \mu_{1} \mu_{2} \kappa^{2}-3 \mu_{2}^{2} \kappa^{4}+12 \delta_{2} \mu_{3} \kappa^{4}\right)
\end{aligned}
$$

with,

$$
\begin{aligned}
& \mu_{1}=\delta_{1} l_{\xi}+\delta_{3} l_{\zeta} \\
& \mu_{2}=3 \delta_{2} l_{\xi}+\delta_{5} l_{\zeta} \\
& \mu_{3}=3 \delta_{2} l_{\xi}^{2}+2 \delta_{5} l_{\zeta} l_{\xi}+\delta_{6} l_{\zeta}^{2}+\delta_{7} l_{\eta}^{2}
\end{aligned}
$$

It is clear from the dispersion relation (43) that there is always instability if $\left(\Upsilon-\Omega^{2}\right)>0$ We can express the instability criterion as

$$
S_{i}>0
$$

with

$$
S_{i}=l_{\eta}^{2}\left[\omega_{c d}^{2}+\sin ^{2} \delta\right]+l_{\zeta}^{2}\left[\omega_{c d}^{2}-\frac{5}{3}\left(\omega_{c d}^{2}+1\right) \tan ^{2} \delta\right]
$$

If this instability criterion $S_{i}>0$ is satisfied, the growth rate $\Gamma=\left(\Upsilon-\Omega^{2}\right)^{1 / 2}$ of the unstable perturbation of these solitary waves is given by

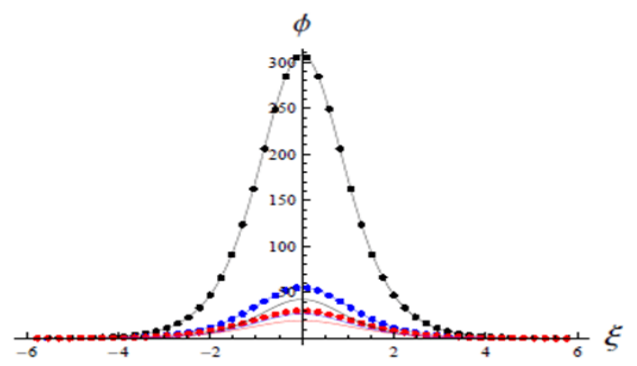

Figure 15: Variation of $\varphi$ with $\xi$ for the values of $\alpha=0.5, \omega_{c d}=0.3, \delta=20^{\circ}, u_{0}=0.1, \mu_{p}=1$, $\mu_{i}=0.4, \mu_{e}=1.2, \sigma_{d}=1, \beta=0.6$ (Black), $\beta=0.7$ (Blue) and $\beta=0.8$ (Red). Here dotted curve represented ultra-relativistic $(\gamma=4 / 3)$ case and solid curve represented non-relativistic $(\gamma=5 / 3$ ) case. 


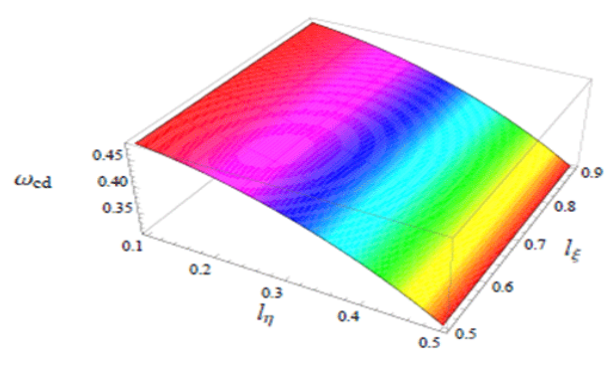

Figure 16: $S_{t}=0$ surface plot showing the variation of $\omega_{c d}$ with $l_{\eta}$ and $l_{\xi}$ for the values of $\mu_{e}=1.2, \mu_{p}=1, \mu_{i}=0.4, \beta=1, \alpha=0.5, \sigma=1$ and $\delta=20^{\circ}$.

$$
\Gamma=\frac{2}{\sqrt{15}} \frac{U_{0}}{\left(\omega_{c d}^{2}+\sin ^{2} \delta\right)} \sqrt{\left(\omega_{c d}^{2}+1\right) S_{i}}
$$

Figure 16 represent $S_{i}=0$ surface plot showing the variation of $\omega_{c d}$ with $l_{\eta}$ and $l_{\xi}$, From this figure we found that $\omega_{c d}$ decreases with increasing the value of $l_{\eta}$ very rapidly and increases slowly with increasing the value of $l_{\xi}$ Figure 17 showing the variation of growth rate $\Gamma$ with $\sigma_{d}$ and $\beta$ We found from this figure that $\Gamma$ decreases with increasing the value of $\sigma_{d}$ and $\beta$ Figure 18 shows the variation of $\Gamma$ with $\mu_{i}$ and $\mu_{e}$ We have found from this figure that $\Gamma$ decreases with increasing the value of $\mu_{e}$ and $\mu_{i}$ Figure 19 has shown the variation of growth rate $\Gamma$ with $\alpha$ and $\beta$ We have seen from the figure that $\Gamma$ increases with increasing the value of $\alpha$.

\section{Findings}

DA SW's have been examined in a collisionless dusty plasma consisting of inertialess ultra-relativistic and non-relativistic electrons and positrons, inertial mobile positive dust particles, inertial Maxwellian ions by deriving K-dV equation using reductive perturbation technique. It has been found that the basic features of such DA solitary waves are significantly modified by the presence degeneracy of adiabaticness of components. The effects of the parametric regimes on solitary wave for both case of unmagnetic and magnetic fields are discussed below:

1. Depending on the value of $\mu_{e}, \mu_{p}$ and $\beta$ the solitary waves can be associated with positive (negative) potentials above (below) the $A=0$ surface for unmagnetized and $P=0$ surface for magnetized plasmas respectively.

2. The amplitude of the DASW's is higher for ultrarelativistic case than non-relativistic one.

3. Magnetic field enhance the amplitude of the SW's for both ultra-relativistic and non-relativistic case.

4. Both amplitude and width of the solitary waves increases with increasing the value of $\alpha, \delta, \sigma_{d}$ and $\mu_{e}$ but decreases with increasing the value of $\mu_{i}, \mu_{p}$ and $\beta$ in the case of unmagnetized as well as magnetized dusty plasmas.
5. Cyclotron frequency $\omega_{c d}$ increases with increasing the value of $l_{\eta}$ and decreasing the value of $l_{\xi}$.

6. The growth rate $\Gamma$ decreases with increasing the value of $\sigma, \beta, \mu_{e}$ and but increases with increasing the value of $\alpha$.

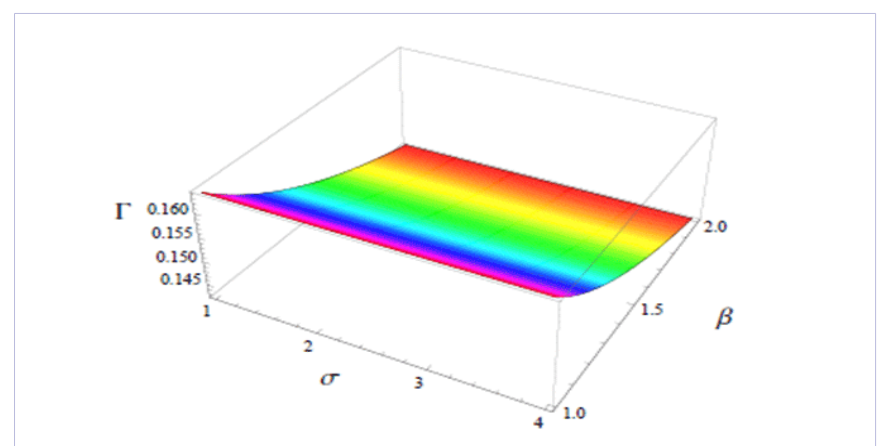

Figure 17: Variation of growth rate ( $\Gamma$ ) with $\sigma$ and $\beta$ for the values of $\mu_{e}=1.2, \mu_{p}=1, \mu_{i}=0.4$ , $\delta=2^{0}, \alpha=0.2, l_{\eta}=0.4, l_{\xi}=0.5, u_{0}=0.1$ and $\omega_{c d}=0.3$.
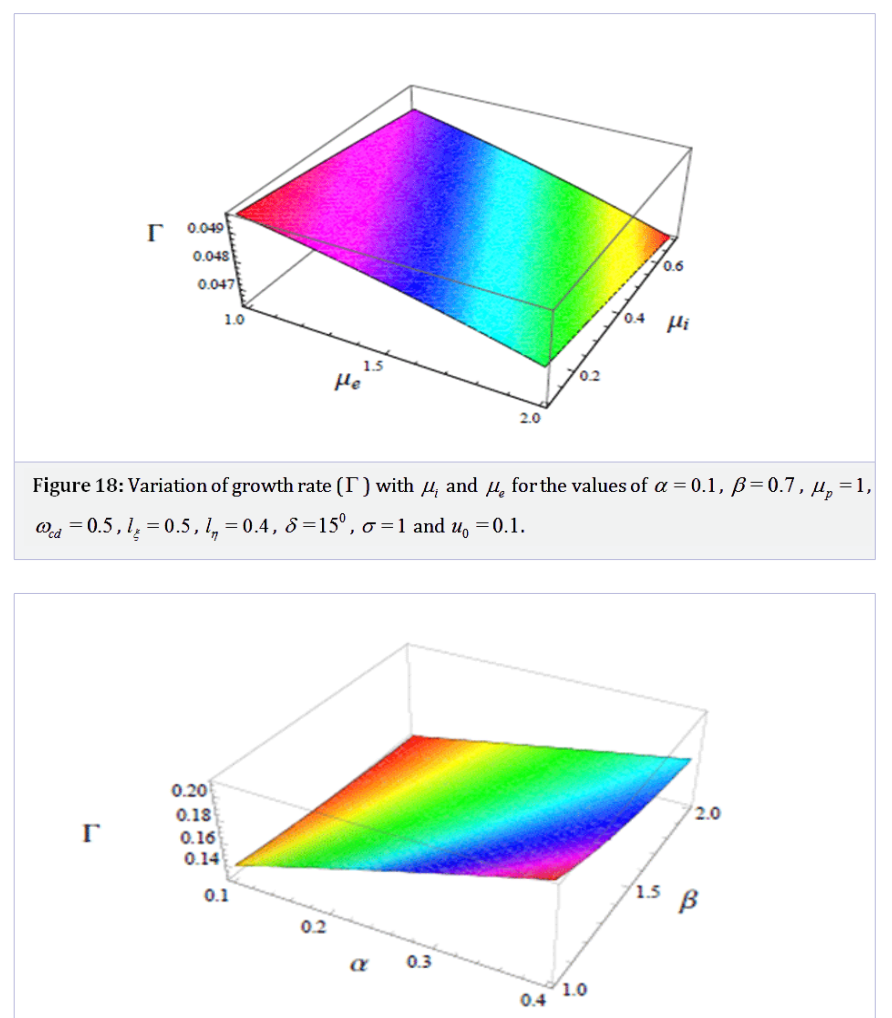

Figure 19: Variation of growth rate $(\Gamma)$ with $l_{\eta}$ and $l_{\xi}$ for the values of $\beta=0.7, \mu_{p}=1$, $\omega_{c d}=0.3, \mu_{e}=1.2, \mu_{i}=0.4, \delta=2^{0}, \sigma=1, l_{\xi}=0.5$ and $u_{0}=0.1$.

\section{Conclusion}

In conclusion we can say that Maxwellian ions can modify the basic properties of the solitary waves significantly. On the other hand the presence of magnetic field effects the amplitude and width of the solitary wave and its instability. The properties of the solitary waves also moderated by the presence the adiabetic pressers in degenerate plasmas. We have shown the variation 
of solitary wave phenomenon and its stabilities criterion as well as its growth rate due to the ultra-relativistic and nonrelativistic degenerate pressure. These theoretical analysis may helpful astrophysicist to understand different critical situations exist in white dwarf and neutron stars; not only that, the other astrophysical objects where degenerate pressure plays a vital role to sustain these. Compering the present analysis to others (non-degenerate objects) one give a prediction on the situation of degenerate astrophysical objects.

The plasma parameters used in the present investigations correspond to white dwarfs though it can also be applied for neutron stars. As the number density of plasma parameters, so as the value of $\beta$, is much higher for neutron stars than white dwarfs; it can be predict that the amplitude of the SW's would be higher. But numerical analysis is essential to view the true fact. We hope we will see the actual variation of wave properties between white dwarf and neutron stars very soon. We hope that our present investigation can helpful for understanding the white dwarfs and neutron stars and give a guideline for then who wants to work in the relevant field.

\section{References}

1. Bliokh PV and Yaroshenko VV. Electrostatic Waves in Saturns Rings. Sov. Astron. 1985;29:330-336.

2. Smith BA, Soderblom L, Batson R, Bridges p, Inge J, Masursky H, et al, A New Look at the Saturn System: The Voyager 2 Images, Science. 1992;215(4532):504-537. DOI: 10.1126/science.215.4532.504.

3. Rao NN, Shukla PK and Yu MY. Dust-acoustic waves in dusty plasma. Plant Space Sci. 1990;38(4):543-546.

4. Roychoudhury R and Mukherjee S. Large-amplitude solitary waves in finite temperature dusty plasma. Phys. Plasmas. 1997;4(6).

5. Sayed F and Mamun AA. Dust-acoustic Korteweg-de Vries solitons in an adiabatic hot dusty plasma. Phys. Plasmas.2007;4(1).

6. Washimi H and Taniuti T. Propagation of Ion-Acoustic Solitary Waves of Small Amplitude, Phys. Rev. Lett. 1996;17:996.

7. Mendoza-Briceño CA, Russel SM and Mamun AA. Large amplitude electrostatic solitary structures in a hot non-thermal dusty plasma. Planet. Space Sci.2000;48(6):599-608

8. Cairns RA, Mamum AA, Bingham R, Boström R, Dendy RO and Nairn CMC, et. al. Electrostatic solitary structures in nonthermal plasmas. Geophys. Res. Lett.1995;22(20):2709-2712. DOI: $10.1029 / 95 \mathrm{GL} 02781$.

9. Bernstein B, Greene JM and Kruskal MD. Exact Nonlinear Plasma Oscillations, Phys. Rev.1957;108:546.
10. Chanrdasekhar S. Introduction to the study of stellar structure. Mon. Not. R. Astron. Soc.1935;170:405

11. Chanrdasekhar S. The Maximum Mass of Ideal White Dwarfs. Astrophys. J.1931;74:81.

12. Mamun AA and Shukla PK. Solitary waves in an ultrarelativistic degenerate dense plasma. Phys. Plasma.2010;17(10).

13. Mamun AA and Shukla PK. Arbitrary amplitude solitary waves and double layers in an ultra-relativistic degenerate dense dusty plasma. Phys. Lett. A.2010;374(41): 4238-4241.

14. Mamun AA, Duha SS and Shukla PK. Multi-dimensional instability of multi-ion acoustic solitary waves in a degenerate magnetized plasma. J Plasma Phys.2011;77(5):617-628.

15. Haider MM, Akter S, Duha SS and Mamun AA. Multi-dimensional instability of electrostatic solitary waves in ultra-relativistic degenerate electron-positron-ion plasmas. Cent. Eur. J. Phys.2012;10(5):1168-1176.

16. Haider MM and Mamun AA. Ion-acoustic solitary waves and their multi-dimensional instability in a magnetized degenerate plasma. Phys. Plasma.2012;19(10)

17. Haider MM. Soliton and Shock Profiles in Electron-positronion Degenerate Plasmas for Both Nonrelativistic and Ultra-Relativistic Limits. Z. Naturforsch.2016;71(12):1131-1137.

18. H. Schamel. Stationary solitary, snoidal and sinusoidal ion acoustic waves. Plasma Phys.1972;14(10):905-924.

19. Schamel H. Analytic BGK modes and their modulational instability. Plasma Physics.1975;13(1):139-145.

20. Witt $\mathrm{E}$ and Lotko $\mathrm{W}$. Ion-acoustic solitary waves in a magnetized plasma with arbitrary electron equation of state. Phys. Fluids.1983; 26(8):2176

21. Rpwlands G. Stability of non-linear plasma waves. J. Plasma Phys.1969;3(4):567-576.

22. Infeld E. On the stability of nonlinear cold plasma waves. J. Plasma Phys.1972;8(1):105-110.

23. Infeld E. Self-focusing of nonlinear ion-acoustic waves and solitons in magnetized plasmas. J. Plasma Phys.1985;33(2):171-182.

24. Infeld $\mathrm{E}$ and Rowlands $\mathrm{G}$. On the stability of nonlinear cold plasma waves. Part 2. J. Plasma Phys.1973;10(2):293-300

25. Laedke EW and Spatschek KH. Growth rates of bending KdV solitons. J. Plasma Phys.1982;28(3):469-484.

26. Mamun AA and Cairns RA. Stability of solitary waves in a magnetized non-thermal plasma. J. Plasma Phys.1996;56(10):175-185. 